\title{
Investigation of the Use of Loudspeaker as a Liner Alternator for Thermoacoustic Application
}

\author{
B.M. Chen, R.H. Jiang \\ Key Laboratory of Distributed Energy Systems of Guangdong Province \\ Department of Energy and Chemical Engineering \\ Dongguan University of Technology \\ China \\ P. Riley \\ The University of Nottingham \\ Nottingham, UK
}

\author{
K. Ho, A. Abakr \\ The University of Nottingham Malaysia Campus \\ Malaysia
}

\begin{abstract}
Thermoacoustic engine technology has been applied to convert heat energy into acoustic energy for the purpose of electricity generation. However, linear alternators are relatively expensive. Recently, SCORE (www.score.uk.com) has successfully developed 22.7 watts of electricity power using a Thermo-Acoustic engine (TAE) with an ordinary audio loudspeaker as a linear alternator. However, the powertransduction efficiency of loudspeakers is usually poor due to their physical properties such as paper cones are weak and fragile, strokes are limited, and their impedances are poorly matched to gas at high pressure. As a matter of fact, it is good to understand the physical properties of loudspeakers so as to understand better of the overall power transduction efficiency. This paper describes a method of measuring a low-cost loudspeaker's Thiele/Small parameter (T/S) for the thermoacoustic engine electrical generation application based on the method proposed by A.N.Thiele and Richard H. Small.
\end{abstract}

Keywords-low-cost; linear alternator; loudspeaker; thermoacoustic; thiele/small parameter

\section{INTRODUCTION}

The thermoacoustic engine's acoustic powered derived from the heat input can be utilized in different ways for different applications [1] [2]. However, generally it can be used for two main purposes: one is to drive coolers or heat pumps, which can be either thermoacoustic coolers (heat pumps) or pulsed-tube coolers [3] [4]. The other is to directly convert the acoustic power to electricity through the electrodynamic transduction mechanism [5] [6] [7]. Usually, flexure bearing-supported linear alternators are an excellent solution due to their high reliability and efficiency. For instance, the well-known travelling wave thermoacoustic electric generator [8] developed by Backhaus group reaches the alternator efficiency (the electric power output over acoustic power absorbed by the alternator) of 0.75 .

However, linear alternators are relatively costly, which limits the advantages of the thermoacoustic heat engines for low-cost energy converting devices [9]. Usually, ordinary audio loudspeakers have an invert working principle as an alternator, it is possible to be considered as an alternative for thermoacoustic electricity generation application where the main driver is the cost of the device, not the power transduction (or even the overall) efficiency. This is particularly true for the wasted heat and solar energy utilization applications, where a low grade thermal energy is abundant and could be considered a limitless source or waste energy. Then the actual efficiency may become a secondary issue. As a matter of fact, it is good to know the Thiele/Small actual parameters of the loudspeaker so as to judge its transducer's real performance rather than the stated basic benchmarks such as size, maximum power rating or average sensitivity. Standard measurement criteria are required to enable manufacturers to publish consistent data for customers. Therefore, commercialize-branded products often come with its data when user purchase. Generally, these branded products are relatively expensive (for a 4 inch woofer, usually cost US\$ 100) partially due to their high testing cost of the consistent data. In this paper, an easy and inexpensive method of measuring the low-cost (US\$ 15) loudspeaker's Thiele/Small parameters is described (no T/S data are provided when purchase).

It is possible to get most of the Thiele/Small parameters from a loudspeaker by just accurately measuring the impedance versus frequency. If this is done twice, one with the driver in open air and one with an added (known) mass if should be fairly easy to obtain most of the data.

\section{DEFINITIONS AND MEASUREMENT METHODOLOGY}

\section{A. Definitions}

TABLE I .DEFINITIONS OF A LOUDSPEAKER' PARAMETERS.

\begin{tabular}{|c|l|c|l|}
\hline$R_{e}$ & $\begin{array}{l}\text { Electrical resistance of } \\
\text { voice coil [Ohm] }\end{array}$ & $C_{m s}$ & $\begin{array}{l}\text { Mechanical compliance } \\
\text { (reciprocal of stiffness) } \\
{[\mathrm{m} / \mathrm{N}]}\end{array}$ \\
\hline$F_{s}$ & $\begin{array}{l}\text { Resonant frequency of } \\
\text { loudspeaker moving } \\
\text { mass in free air [Hz] }\end{array}$ & $V_{a s}$ & $\begin{array}{l}\text { Equivalent air volume of } \\
\text { moving mass suspension } \\
\text { [Liters] }\end{array}$ \\
\hline$Q_{e s}$ & $\begin{array}{l}\text { Electrical Q-factor of } \\
\text { loudspeaker }\end{array}$ & $B L$ & Magnetic strength [Tm] \\
\hline$Q_{m s}$ & $\begin{array}{l}\text { Mechanical Q-factor of } \\
\text { loudspeaker }\end{array}$ & $X_{m a}$ & $\begin{array}{l}\text { Maximum peak linear } \\
\text { excursion of loudspeaker } \\
\text { [mm] }\end{array}$ \\
\hline$Q_{t s}$ & $\begin{array}{l}\text { Total Q-factor of } \\
\text { loudspeaker }\end{array}$ & $L_{e}$ & Voice coil inductance [mH] \\
\hline
\end{tabular}


A typical example of the impedance curve for a loudspeaker is shown on Figure. 1. Resonance causes a large increase in impedance and at some higher frequency, the inductance (or semi-inductance) of the voice coil causes the impedance to rise again. The section for the primary measurements must be within the "linear" region of the impedance curve. In the example (Figure. 1), resonance is at $61 \mathrm{~Hz}$, and the linear region ranges from about $310 \mathrm{~Hz}$ to $850 \mathrm{~Hz}$.

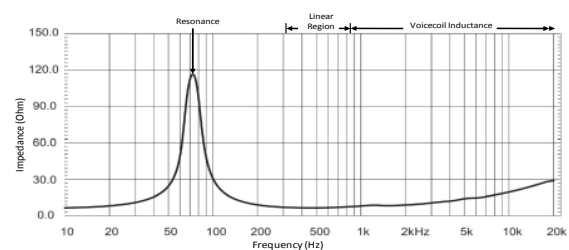

FIGURE I. .TYPICAL EXAMPLE OF THE LOUDSPEAKER IMPEDANCE CURVE.

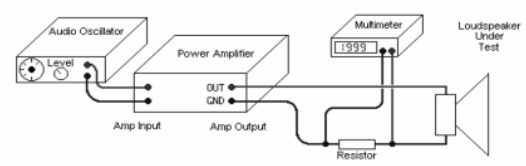

FIGURE II. MEASURING THE MAIN SPEAKER PARAMETER.

It can be understood that at resonance, the speaker impedance is pure resistance. As the frequency increasing towards resonance, the impedance characteristic is inductive. Beyond resonances as impedance falls, the impedance characteristic is capacitive. Within the "linear" region, the impedance is again resistive, but at slightly below the speaker's nominal impedance (nominal impedance is usually taken as an average value over the usable frequency range). At the frequency where the inductance of the voice coil becomes significant, impedance rises, and is progressively more inductive as the frequency rises. It is common to add a compensation network to maintain an overall resistive characteristic at these higher frequencies, so that the performance of this passive crossover network is not compromised. However, this is not necessary with an active crossover.

1) Measurement methodology: In this method outlined below, the following parameters of loudspeakers are measured and calculated: $R_{e}, F_{s}, Q_{e s}, Q_{m s}, Q_{t s}, V_{a s}, B L$ and $X_{\text {max }}$, the definitions of the parameters are shown in Table 1.

The loudspeaker driver is suspended in free space, with no obstructions or interfering surfaces nearby. The resistance is measured across the speaker terminals using the Multimeter to obtain $R_{e}$. The exact resistance of the $10 \mathrm{ohm}$ source on the Rheostat is measure using Multimeter, this is $R_{s}$. The circuit is connected as shown in Figure. 2, and the frequency input to the speaker is set to the range of $150 \mathrm{~Hz}$ (or 2-3 octaves above resonance).

The output of the amplifier is set to between $0.5 \mathrm{~V}$ and $1.0 \mathrm{~V}$ (this is $V_{s}$ ). It may need to experiment with different voltages, depending on the accuracy of the current readings. It is not advisable to use higher voltages, as the speaker may be driven outside its linear range, which ruins the validity of the measurements. The parameters being measured are "small signal", and it essential that a small signal is actually used.

Therefore, the reference speaker current, $I_{s}$ which is equal to the meter reading $\left(V_{s}\right)$ at $150 \mathrm{~Hz}$ divided by the value of $R_{s}$, to make sure that a reasonable current to work with. That the speaker is then checked nowhere near resonance, by changing the output frequency by $50 \mathrm{~Hz}$ or so in either direction then measure the voltage across the speaker. It should not be changed by any appreciable amount.

The traditional way to measure $Q$ is to measure the bandwidth between the $-3 \mathrm{~dB}$ frequencies, then divide the resonator frequency by the bandwidth. However, this method is more suitable for low- $Q$ drivers. Small's has stated that $f l$ and $f 2$ are the frequencies where the drive unit impedance is $\sqrt{r 0} \times R_{e}$ [10]. Likewise, $r O=\left(R_{e}+R_{e s}\right) / R_{e},\left(R_{e}+R_{e s}\right)$ being the impedance at $F_{s}$. It has chosen $\sqrt{r} 0 \times R_{e}$ to simplify the calculation for $Q_{m s}$ and $Q_{e s}$. Many of the methods described elsewhere rely on a more complex formula that use $-6 \mathrm{~dB}$ or even $-9 \mathrm{~dB}$ as the reference point to determine $Q$. This makes the measurement accuracy slightly less critical. In this measurement, the $-6 \mathrm{~dB}$ method is used, which gives a reasonable compromise between ease of measurement and accuracy.

First, the resonant frequency $\left(F_{s}\right)$ is measured. The output frequency is adjusted until the voltage across the resistor $\left(V_{m}\right)$ reaches a null (minimum value). Without changing anything, the frequency and voltage across the resistor is carefully measured. By performing the following calculation:

$$
\begin{aligned}
& \text { Speaker Current: } I_{m}=\frac{V_{m}}{R_{s}} \\
& \text { Resonance impedance: } R_{m}=\frac{\left(V_{s}-V_{m}\right)}{I_{m}} \\
& \text { r0 (reference value): } r 0=\frac{I_{s}}{I_{m}} \\
& \text {-6dB current: } I_{r}=\sqrt{I_{m} \times I_{s}} \\
& \text {-6dB voltage: } V_{r}=I_{r} \times R_{s}
\end{aligned}
$$

The measurement for $\mathrm{Fl}$ and $\mathrm{Fh}$ is then completed, for which the voltage across the source resistor is equal to $\mathrm{Vr}$, and as a sanity check to ensure that the calculations and measurements are accurate, the resonant frequency is calculated based on these last two measurements by performing the this calculation:

$$
\sqrt{F l \times F h}=F_{s}
$$

If the above check out different is less than $2 \mathrm{~Hz}$, then Qes, Qms and Qts can be calculated as follows: Mechanical Qfactor: 


$$
Q_{m s}=\frac{F_{s} \times \sqrt{r 0}}{(F h-F l)} ;
$$

Electrical Q-factor:

$$
Q_{e s}=\frac{Q_{m s}}{(r 0-1)} \times \frac{R_{e}}{\left(R_{e}+R_{s}\right)}
$$

Total Q-factor:

$$
Q_{t s}=Q_{m s} \times \frac{Q_{e s}}{\left(Q_{m s}+Q_{e s}\right)}
$$

To measure Vas, simply applying mass (known) on the cone so as to calculate the moving mass of the cone perhaps is the most convenient way to determine the value of Vas. Typically modeling clay or Blu-Tak is simply stuck to the cone close to the voice coil, and the change of resonant frequency allows us to determine the moving mass of the cone. By doing this, the Vas then can be calculated. Simply add a suitable mass (M1) to the cone and re-measure the resonant frequency based on the described method for measuring the Fs. This becomes Fs1. Generally, for speakers less than $200 \mathrm{~mm}$ (8"), use 5 grams. However, it may need to add more if the mass chosen does not reduce resonance by at least $10 \%$. The mass must be measured accurately, so a precision scale (accurate to at least $0.1 \mathrm{~g}$ ) is essential.

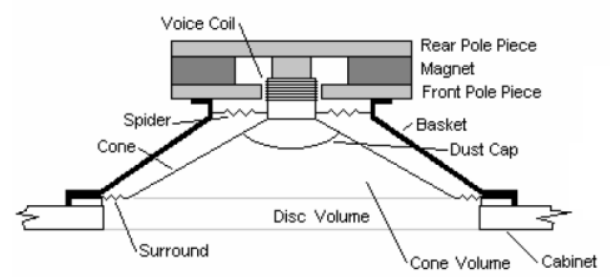

FIGURE III. DETERMINING THE VOLUME OF THE CONE.

Other than this, the effective cone diameter needs to be measured. This is generally taken as a measurement that includes half the surround (Referring to Figure. 3). It is good to use centimeters in the measurement, and then divide by 2 to get the radius for this calculation.

$$
A=\pi \times r^{2}
$$

Calculating cone mass:

$$
M=\frac{M_{1}}{\left.\left(\sqrt{\frac{F_{S}}{F_{S}^{1}}}\right)-1\right)}
$$

$$
\begin{aligned}
\text { Next: Determine } & C_{m s}, \\
C_{m s} & =\frac{1}{\left[\left(2 \times \pi \times F_{S}\right) 2 \times M\right]}
\end{aligned}
$$

and

$$
V_{a s}=C_{m s} \times d \times c^{2} \times A^{2}
$$

where, $d$ is the density of air $(0.001204 \mathrm{~g} / \mathrm{ml}) . c$ is the speed of sound $(345 \mathrm{~m} / \mathrm{s})$.

There are two ways to measure the magnetic strength of the motor structure, BL, One static and one dynamic. This is measurement of the motor strength of a speaker, expressed in Tesla meters. A high BL figure indicates a very strong transducer that moves the cone with authority. Think of this as how good a weightlifter the transducer is. A measurement mass is applied to the cone forcing it back while the current required for the motor to force the mass back is measured. The formula is mass in grams divided by the current in amperes. $B L i=F$, so, $B L=F / i$, where $F$ is the force which is measured at the null position of the cone, the cone must not move away from its central position. $i$ is the current.

In this measurement, the loudspeaker is placed on the level table with the cone face up. A straight edge rule is putting across the top, and a depth gauge to the dome is used for measuring the movement of the cone. A sufficiency mass is then added into the cone, and apply a current using a current limited power supply until the cone returns to its null position. Generally, for a $4 \mathrm{ohm}$ speaker with $B L$ of $10,1 \mathrm{amp}$ should give a $7 \mathrm{~N}(700 \mathrm{~g})$ forces.

Le is the voice coil inductance measured in millihenries $(\mathrm{mH})$. The industry standard is to measure inductance at $1000 \mathrm{~Hz}$. As frequency getting higher there will be a rise in impedance above $R e$. This is because the voice coil is acting as an inductor. In this measurement, it will be sufficient for our purpose and not far off to measure the voice coil inductance by direct reading using an inductance meter.

2) Measurement result: The testing loudspeaker for this measurement is the low-cost (US\$ 15) Brother ${ }^{\mathrm{TM}}$ HT-655 loudspeaker, the Thiele/Small parameters of this loudspeaker were measured/calculated using the method described, and the measurement results are showing below:

$$
\begin{array}{ll}
R e=3.80 \Omega, & V s=0.65 \mathrm{~V}, \quad V m=0.165 \mathrm{~V} ; \\
R s=10 \Omega, & F s=65 \mathrm{~Hz},
\end{array}
$$

Calculating $V r$ :

$\begin{array}{ll}\text { Reference speaker } & \text { r0 } \quad \text { (reference } \\ \text { current: } I_{s}=0.065 \mathrm{~A} & \text { value): } r 0=3.93 \\ \text { Speaker Current: } & -6 \mathrm{~dB} \quad \text { current: } \\ I_{m}=0.0165 \mathrm{~A} & I_{r}=0.033 \mathrm{~A} \\ \text { Resonance } & -6 \mathrm{~dB} \quad \text { voltage: } \\ \text { impedance: } & V_{r}=0.330 \mathrm{~A} \\ R_{m}=29.30 \Omega & \end{array}$

Calculating Qes, Qms, and Qts:

From the measurement, the value of $F l$ and $F h$ is obtained,

$F l=60 \mathrm{~Hz}$ and $F h=76 \mathrm{~Hz}$;

Mechanical Q-factor: $Q_{m s}=6.443$;

Electrical Q-factor: $Q_{e s}=0.605$; 
Total Q-factor: $Q_{t s}=0.553$.

Calculating $V_{a s}$ : The diameter of the loudspeaker is measured, $d=12.74 \mathrm{~cm}$, the mass of the object, $M_{l}=5.00 \mathrm{~g}$ and the new resonant frequency, $F_{s}{ }^{l}=56 \mathrm{~Hz}$ is obtained. Therefore, effective area, $A=127.48 \mathrm{~cm}^{2}$, cone mass, $M=14.40 \mathrm{~g}$, mechanical compliance (reciprocal of stiffness), $C_{m s}=4.16 \times 10^{-}$ ${ }^{7}$, equivalent air compliance, $V_{a s}=9.69 \mathrm{~L}$.

For applying $1000 \mathrm{~g}$ mass on the loudspeaker cone, $i=1.48 \mathrm{~A}$ was obtained, so, $B L=6.63 . L_{e}$ is measured to be $33.0 \mathrm{mH}$. And finally, $X_{\max }=5.58 \mathrm{~mm}$ is measured and calculated.

\section{SUMMARY}

In this paper, most of the Thiele/Small parameters of a low-cost BrotherTM loudspeaker are measured and calculated based on the method described. The results have been also compared with known parameters; it is found that the results obtained are appropriate.

\section{ACKNOWLEDGEMENTS}

This work was supported by the China National Natural Science Foundation Project (No. 51406036).

\section{REFERENCES}

[1] G. W. Swift, Thermoacoustics: A unifying perspective for some engines and refrigerators: Acoustical Society of America, 2002.

[2] E. Luo, W. Dai, Y. Zhang, and H. Ling, "Experimental investigation of a thermoacoustic-Stirling refrigerator driven by a thermoacoustic-Stirling heat engine," Ultrasonics, vol. 44, pp. e1531-e1533, 2006.

[3] W. Dai, E. Luo, Y. Zhang, and H. Ling, "Detailed study of a traveling wave thermoacoustic refrigerator driven by a traveling wave thermoacoustic engine," The Journal of the Acoustical Society of America, vol. 119, p. 2686, 2006.

[4] M. Tijani, J. Zeegers, and A. De Waele, "Design of thermoacoustic refrigerators," Cryogenics, vol. 42, pp. 49-57, 2002.

[5] E. C. Luo, Z. H. Wu, W. Dai, S. F. Li, and Y. Zhou, "A 100 W-class traveling-wave thermoacoustic electricity generator," Chinese Science Bulletin, vol. 53, pp. 1453-1456, 2008.

[6] B.M. Chen, P.H. Riley, Y.A. Abakr, K. Pullen, D.B. Hann, C.M. Johnson, Design and development of a low-cost, electricity-generating cooking Score-Stove ${ }^{\mathrm{TM}}$, Proceedings of the Institution of Mechanical Engineers, Part A: Journal of Power and Energy, 227(7), 803-813, 2013.

[7] B.M. Chen, Y.A. Abakr, P.H. Riley, D.B. Hann, Development of thermoacoustic engine operating by waste heat from cooking stove, American Institute of Physics Conference Proceedings, 1440, 532-540, 2011.

[8] S. Backhaus, et al., "Traveling-wave thermoacoustic electric generator," Applied Physics Letters, vol. 85, p. 1085, 2004.

[9] G. W. Swift and S. L. Garrett, "Thermoacoustics: A unifying perspective for some engines and refrigerators," The Journal of the Acoustical Society of America, vol. 113, p. 2379, 2003.

[10] R. H. Small, "Total Difference Frequency Distortion: Practical Measurements," 1984, p. 464. 